\title{
Exposure therapy and sertraline in social phobia:
}

\section{I-year follow-up of a randomised controlled trial}

\author{
TONE TANGEN HAUG, SVEIN BLOMHOFF, KERSTIN HELLSTR $\varnothing M$, \\ INGAR HOLME, MATS HUMBLE, HANS PETTER MADSBU and JAN EGIL WOLD
}

\section{Background Maintenance of treatment effect is important for the choice of treatment for social phobia.}

\begin{abstract}
Aims To examine the effect of exposure therapy and sertraline 28 weeks after cessation of medical treatment.
\end{abstract}

\begin{abstract}
Method In this study 375 patients with social phobia were randomised to treatment with sertraline or placebo for

24 weeks, with or without the addition of exposure therapy. Fifty-two weeks after inclusion, 328 patients were evaluated by the same psychometric tests as at baseline and the end of treatment ( 24 weeks).
\end{abstract}

Results The exposure therapy group and the placebo group had a further improvement in scores on social phobia during follow-up: mean change in the Clinical Global Impression - Social Phobia overall severity score was 0.45 (95\% Cl 0.16-0.65, $P<0.0$ I) for the exposure group, and 0.25 (95\% Cl 0.00-0.48, $P<0.05$ ) for the placebo group. At week 52 the sertraline plus exposure group and the sertralinealone group had a significant deterioration on the 36 -item Short Form Health Survey compared with exposure alone.

\section{Conclusions Exposure therapyalone yielded a fur ther improvement during follow-up, whereas exposure therapy combined with sertraline and sertraline alone showed a tendency towards deterioration after the completion of treatment.}

Declaration of interest Funding was provided by Pfizer, Inc.
The short-term effect of treatment of social phobia has been demonstrated in several studies, and both cognitive-behavioural treatments and pharmacological interventions have given positive findings (Mattick et al, 1989; Gelernter et al, 1991; Liebowitz et al, 1992; Versiani et al, 1992; Davidson et al, 1993; Heimberg et al, 1993; Van Vlet et al, 1994; Katzelnick et al, 1995; Taylor, 1996; Stein et al, 1998). The studies vary widely in terms of outcome measures, type of control groups, sample selections and whether treatment is offered individually or in groups, making it impossible to draw meaningful comparisons between studies. A few studies have compared the effect of psychological treatment with the effect of medication (Turner et al, 1994; Heimberg et al, 1998), but it has been difficult to identify treatments that are clearly superior to others. Which form of treatment is chosen will therefore mainly depend on the patient's preferences and the availability of services. However, the maintenance of treatment effects after cessation of active treatment is also important in this decision.

In this study we examine the effect on generalised social phobia of 24 weeks of treatment with sertraline, with or without the addition of exposure therapy, 1 year after the start of treatment.

\section{METHOD}

People aged 18-65 years with generalised social phobia according to DSM-IV criteria (American Psychiatric Association, 1994) of at least 1 year's duration and rated as moderately ill (a score of at least 4) on the overall severity item of the severity sub-scale of the Clinical Global Impression - Social Phobia scale (CGI-SP, range 1-7, Davidson et al, 1993) were eligible for inclusion in the study. Participants were consecutively recruited from people seeking medical care at 41 different primary care centres in Norway and Sweden $(n=289)$ and from advertisements in newspapers and other media $(n=159)$. Three hundred and seventy-five patients were randomly assigned by a computer to receive doubleblind sertraline or placebo in blocks of eight, so that four patients in each block were randomised to each treatment. No other stratification factor was used. Each block was assigned to a specific general practitioner. In both the sertraline and the placebo groups half of the patients were randomly allocated to exposure therapy or to general medical care only. A separate randomisation list was made for exposure or non-exposure treatment. Sealed envelopes for allocations from this list were kept by the investigators and opened after inclusion of the patient into the study. During this procedure equal numbers of participants were assigned to each treatment option in each block. Tablets were packaged and numbered by the sponsor and personally delivered to each investigator. Participants were evaluated at week 24 (post-treatment, $n=346$ ) and at week 52 (follow-up, $n=328$; Fig. 1). Of the original 375 participants, 228 were female and 147 were male; their mean age was 39.8 (s.d. $=10.4)$ years. Mean age at symptom onset was 16.2 (s.d.=9.1) years and the mean duration of illness was 23.6 (s.d.= 12.2) years. Patients with comorbid dysthymia or specific phobias were allowed to enter the study; those with panic disorder with onset before social phobia or any other current anxiety or major depressive disorder, substance misuse or an eating disorder were not eligible. In addition, patients with a lifetime history of bipolar disorder or psychosis were excluded. A comorbid psychiatric disorder was diagnosed in $133(35 \%)$ patients; $101(27 \%)$ had phobic anxiety disorder, $6(1.6 \%)$ panic disorder, $6(1.6 \%)$ dysthymia and 20 $(5.3 \%)$ other diagnoses.

All patients were scheduled for nine meetings with the investigator during the first 16 weeks of treatment and a final efficacy assessment after 24 weeks. The patients were randomised to four treatment groups and treated by general practitioners for 24 weeks with sertraline or a pill placebo, combined with 12 weeks of exposure therapy or of only general medical care. Exposure therapy was given in eight sessions for the first 12 weeks of treatment. Each of the sessions had an estimated duration of 15-20 min. In the first sessions, main problem areas were identified and 


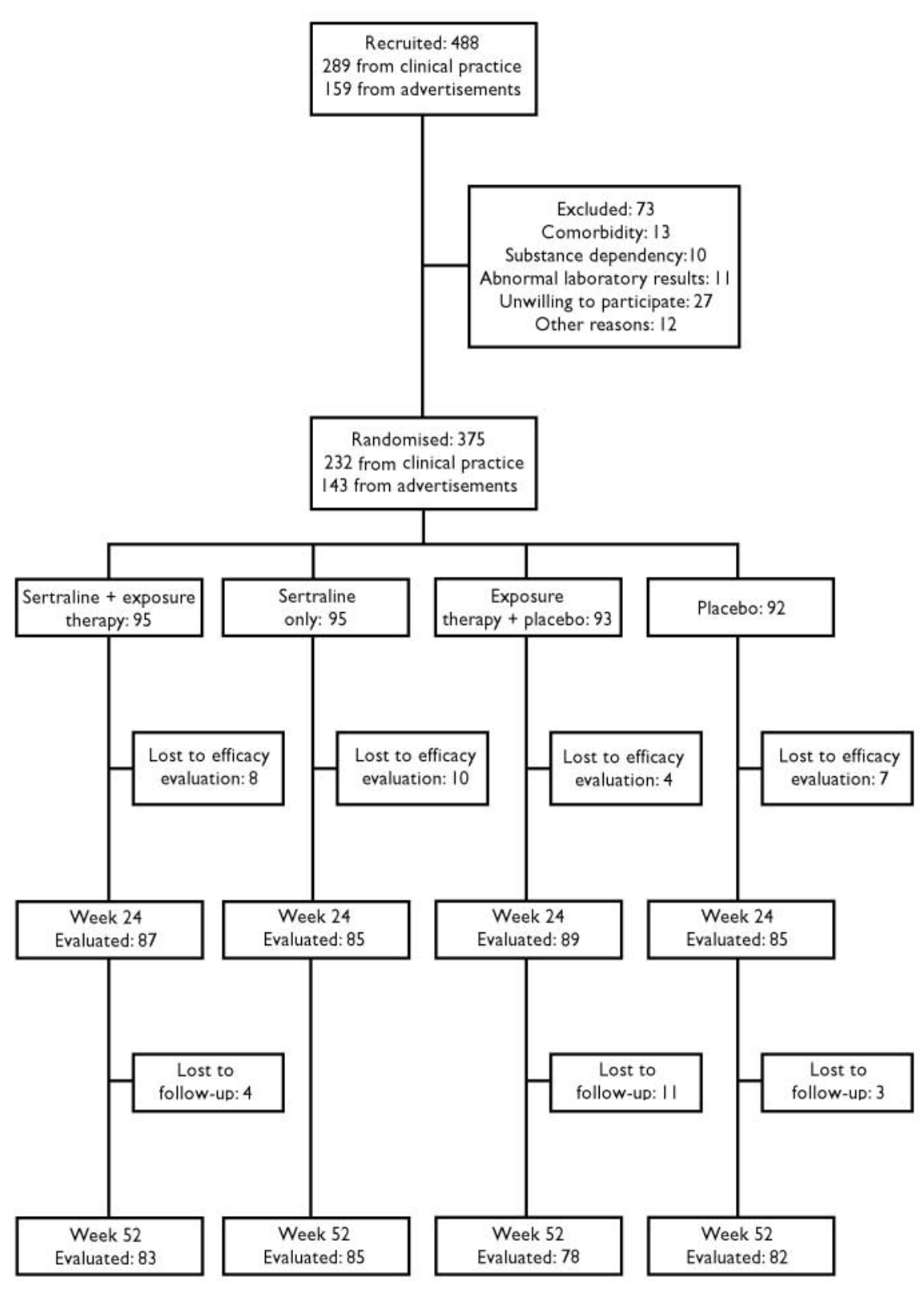

Fig. I Trial profile.

agreement was reached about homework assignments. In the remaining sessions, the patients were instructed to gradually expose themselves to feared situations, and to keep exposure homework diaries. Details of the exposure therapy have been published elsewhere (Haug et al, 2000). At week 24, patients treated with sertraline were significantly more improved than those who did not receive sertraline $\left(\chi^{2}=12.53, \quad P<0.001 ; \quad O R=0.534,95 \%\right.$ CI $0.347-0.835)$. No significant difference was observed between those who received exposure therapy and those who did not $\left(\chi^{2}=2.18, \quad P=0.140 ; \quad\right.$ OR $=0.732, \quad 95 \%$ CI $0.475-1.134)$. In the pairwise comparisons, combined sertraline and exposure clinical judgement of the general practitioner. At week 52 the participants attended an interview and filled in the same questionnaires used for assessment at baseline and at the completion of therapy (24 weeks).

\section{Instruments}

The Mini International Neuropsychiatric Interview (MINI-R; Sheehan et al, 1994) was used to assess DSM-IV psychiatric diagnoses. The Clinical Global Impression Severity Scale (CGI-SP; Liebowitz, 1992) the Social Phobia Scale (SPS; Mattick \& Clark, 1998), the Fear of Negative Evaluation (FNE) scale (Watson \& Friend, 1969) and the Marks Fear Questionnaire (MFQ, Marks \& Matthews, 1979) assessed the degree of social phobia. The Sheehan Disability Inventory (SDI; Leon et al, 1992) and the mental health sub-scale of the MOS 36-item Short Form Health Survey (SF-36; McHorney et al, 1993) assessed daily functioning. The MontgomeryÅsberg Depression Rating Scale (MADRS; Montgomery \& Åsberg, 1979) assessed degree of depression. Patients were also asked about their employment, sick leave and medical symptoms during the preceding year.

\section{Statistical procedures}

The program SAS version 6.12 for Windows (SAS Institute, 1997) was employed in all analyses. All efficacy analyses were on the intention-to-treat patient population: this population was defined as those who received at least one dose of medication and at least one efficacy evaluation post-baseline. All statistical tests were two-tailed with $\alpha=0.05$. Sample size calculation was based on an estimated $20 \%$ difference between active drug and placebo. This required a sample size of at least 340 patients to detect a significant difference if $\beta=0.10$ and the drop-out rate is $35 \%$. This procedure made the study primarily powered for analyses of sertraline $v$. non-sertraline and exposure $v$. nonexposure, but allowed also pairwise comparisons between the treatment groups. In the latter analyses, however, the power was reduced and the risk of false-negative results increased. Repeated-measures analysis of covariance for each scale measurement at 24 weeks and 52 weeks was done to test differences between treatment groups globally, adjusted for baseline values at week 0 . Multiple ordinal logistic 
Table I Change in scores on the Clinical Global Impression - Social Phobia (CGI-SP) severity sub-scale and the Social Phobia Scale (SPS) from week 24 to week 52

\begin{tabular}{lllll}
\hline Scale & \multicolumn{3}{c}{ Change in score', mean $(95 \% \mathrm{Cl})$} \\
\cline { 2 - 6 } & \multicolumn{1}{c}{ Placebo $(n=82)$} & Sertraline+exposure $(n=83)$ & Sertraline $(n=85)$ & Exposure+placebo $(n=78)$ \\
\hline CGI-SP severity & & & & \\
Anxiety attacks & $0.26(-0.02$ to 0.50$)$ & $-0.01(-0.23$ to 0.21$)$ & $-0.05(-0.28$ to 1.19$)$ & $0.14(-0.14$ to 0.39$)$ \\
Avoidance & $0.22(-0.04$ to 0.47$)$ & $-0.05(-0.31$ to 0.21$)$ & $0.05(-0.25$ to 0.30$)$ & $0.17(-0.10$ to 0.45$)$ \\
Performance anxiety & $0.36 *(0.05$ to 0.56$)$ & $-0.04(-0.24$ to 0.15$)$ & $-0.13(-0.37$ to 0.13$)$ & $0.14(-0.12$ to 0.40$)$ \\
Disability & $0.32^{* *}(0.08$ to 0.53$)$ & $-0.05(-0.27$ to 0.18$)$ & $0.12(-0.19$ to 0.36$)$ & $0.32 * *(0.06$ to 0.52$)$ \\
Overall severity & $0.25 *(0.00$ to 0.48$)$ & $0.00(-0.23$ to 0.23$)$ & $0.10(-0.17$ to 0.33$)$ & $0.45 * *(0.16$ to 0.65$)$ \\
SPS & $1.66(-0.61$ to 3.93$)$ & $0.42(-1.42$ to 2.25$)$ & $-0.18(-2.18$ to 1.82$)$ & $3.86 * *(1.27$ to 5.64$)$ \\
\hline
\end{tabular}

CGI to SP sub-scale range I to 7, SPS range 20 to 100

I. Negative values indicate deterioration.

$* P<0.05 ; * * P<0.01$

regression was also used to identify any statistical interaction between treatment groups on response. Pairwise comparisons for changes from week 24 to week 52, adjusted for baseline, were made between each of the three active-treatment groups and the placebo-only group. At each time point ( 24 weeks and 52 weeks) in the time point analyses all groups were compared pairwise, with Bonferroni $P$-value adjustments for each scale analysed.

\section{RESULTS}

Of the 375 patients assessed at baseline, 346 had a post-therapy assessment at week 24 , and 328 a follow-up assessment at week 52 . Only 18 patients were lost to follow-up between week 24 and week 52. The main reasons for patients dropping out were the lack of perceived need for further treatment, events unrelated to treatment such as moving to another area, or unknown reasons (Fig. 1).

\section{Changes in psychometric scores from baseline to week 52}

All four study groups had a significant reduction in scores on CGI-SP (all subscales), SPS, Brief Social Phobia Scale (all sub-scales; Davidson et al, 1991), MFQ and FNE $(P<0.001)$ from baseline to week 52 . There were also significant reductions in MADRS score and in scores on the SDI and SF-36.

\section{Changes in psychometric scores from week 24 to week 52}

Participants who had been treated with exposure alone had a significant improvement in scores on the CGI-SP overall severity sub-scale (mean change $0.45,95 \%$ CI $0.16-0.65, P<0.01)$ and disability subscale (mean change 0.32 , 95\% CI $0.06-$ $0.52, P<0.01$ ), and on the SPS (mean change $3.86,95 \%$ CI $1.27-5.64, P<0.01$ ) during follow-up. The placebo-only group also had a significant improvement on the CGI-SP overall severity sub-scale (mean change 0.25 , 95\% CI $0.00-0.48$, $P<0.05$ ), disability sub-scale (mean change $0.32,95 \%$ CI $0.08-0.53, P<0.01)$ and performance sub-scales (mean change $0.36,95 \%$ CI $0.05-0.56, P<0.05)$. For the sertraline plus exposure and the sertraline-alone groups there was a slight deterioration in scores on most of the CGI sub-scales and on SPS, but the changes were not significant (Table 1, Fig. 2).

The exposure-alone group had also a significant improvement on the fear and avoidance sub-scales of the Brief Social Phobia Scale (mean changes 1.01, 95\% CI $0.06-1.96, P<0.05$, and $1.07,95 \%$ CI $0.01-2.14, P<0.05$, respectively), MFQ (mean change $3.46,95 \%$ CI $1.27-5.64$,

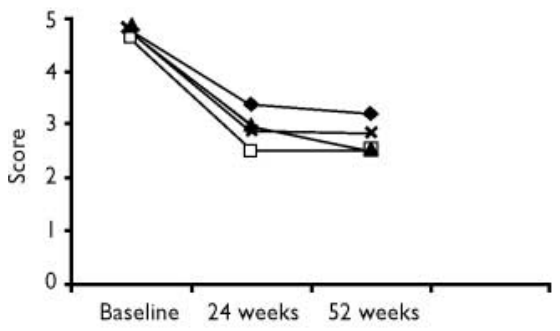

Fig. 2 Mean scores on the Clinical Global Impression - Social Phobia overall severity sub-scale at baseline week 24 and week 52 (range 0-7).

๑, placebo; $\square$, sertraline + exposure; $\boldsymbol{\Delta}$, exposure; $\mathbf{x}$, sertraline.
$P<0.01$ ), FNE (mean change $2.36,95 \%$ CI 0.97-3.77, $P<0.01$ ) and SF-36 (mean change $1.34,95 \%$ CI $0.89-1.85, P<0.01$ ) between week 24 and week 52. The placebo-only group had a significant improvement on the Brief Social Phobia Scale fear sub-scale (mean change 1.01, 95\% CI 0.03-2.00, $P<0.05$ ), MFQ (mean change $3.48, \quad 95 \%$ CI $1.12-5.83$, $P<0.01$ ), FNE (mean change $1.29,95 \%$ CI $0.92-2.65, P<0.05)$, SDI work subscale (mean change 1.07 , 95\% CI 0.44 $1.69, P<0.01$ ), SDI social sub-scale (mean change $0.91,95 \%$ CI $0.27-1.54, P<0.05$ ) and the SF-36 (mean change 1.51, 95\% CI 1.01-2.45, $P<0.01)$. For the sertraline plus exposure and the sertraline-alone groups there was a deterioration in scores on SF-36 between week 24 and week 52 (mean change $-0.89,95 \% \mathrm{CI}-1.35$ to $1.34, P<0.05 ;-1.40,95 \% \mathrm{CI}-1.90$ to $1.92, P<0.01$, respectively). Other changes were not significant (Table 2, Figs 3 and 4).

Compared with the placebo group, the sertraline-alone group were found to have a significant deterioration on the CGI-SP performance anxiety sub-scale (mean difference in change $0.54,95 \% \mathrm{CI}$ 0.13-0.96, $P=0.05$ ), MFQ (mean difference in change $4.84,95 \%$ CI $0.95-8.74$, $P=0.01$ ), FNE (mean difference in change 2.34, 95\% CI $0.15-4.54, P=0.03)$ and SF-36 (mean difference in change -2.92 , $95 \% \mathrm{CI}-4.67$ to $-1.17, P<0.01)$. There was also a significant deterioration in sertraline compared with exposure on MFQ (mean difference in change $4.10,95 \%$ CI $0.17-8.03, P=0.03$ ), FNE (mean difference in change $3.00,95 \%$ CI $0.78-5.22$, $P<0.01$ ) and SF-36 (mean difference in 
Table 2 Change in scores' on the Brief Social Phobia Scale (BSPS), the Fear of Negative Evaluation (FNE) scale, the Marks Fear Questionnaire (MFQ), the Sheehan Disability Inventory (SDI), and the mental health sub-scale of the 36-item Short Form Health Survey (SF-36) from week 24 to week 52 ( $n=328$ )

\begin{tabular}{|c|c|c|c|c|}
\hline \multirow[t]{2}{*}{ Scale } & \multicolumn{4}{|c|}{ Change in score ${ }^{2}$, mean $(95 \% \mathrm{Cl})$} \\
\hline & Placebo $(n=82)$ & Sertraline+exposure $(n=83)$ & Sertraline $(n=85)$ & Exposure+placebo $(n=78)$ \\
\hline \multicolumn{5}{|l|}{ BSPS } \\
\hline Fear & $1.01 *(0.03$ to 2.00$)$ & $0.08(-0.84$ to $\mathrm{I} .00)$ & $0.2 \mathrm{I}(-0.84$ to $\mathrm{I} .27)$ & $1.01 *(0.06$ to 1.96$)$ \\
\hline Somatic reaction & $0.06(-0.54$ to 0.66$)$ & $-0.40(-0.93$ to 0.13$)$ & $0.11(-0.55$ to 0.76$)$ & $0.05(-0.44$ to 0.54$)$ \\
\hline Avoidance & $0.74(-0.24$ to I.7I) & $0.09(-0.77$ to 0.94$)$ & $0.68(-0.31$ to 1.68$)$ & $1.07 *(0.01$ to 2.14$)$ \\
\hline MFQ & $3.48 * *(1.12$ to 5.83$)$ & $0.17(-1.80$ to 2.14$)$ & $-1.27(-3.57$ to 1.02$)$ & $3.46 * *(1.27$ to 5.64$)$ \\
\hline FNE & $1.29 *(0.02$ to 2.56$)$ & $0.33(-0.96$ to $\mathrm{I} .62)$ & $-0.34(-1.49$ to $0.8 \mathrm{I})$ & $2.36 * *(0.96$ to 3.77$)$ \\
\hline \multicolumn{5}{|l|}{ SDI } \\
\hline Work & $1.07^{* *}(0.44$ to $\mathrm{I} .69)$ & $-0.03(-0.51$ to 0.46$)$ & $-0.11(-0.57$ to 0.34$)$ & $0.38(-0.29$ to $\mathrm{I} .05)$ \\
\hline Social & $0.91 *(0.27$ to 1.54$)$ & $-0.18(-0.68$ to 0.31$)$ & $0.08(-0.44$ to 0.59$)$ & $0.64(-0.08$ to 1.36$)$ \\
\hline Family & $0.46(-0.10$ to 1.02$)$ & $-0.09(-0.45$ to 0.26$)$ & $-0.05(-0.50$ to 0.40$)$ & $0.16(-0.31$ to 0.63$)$ \\
\hline SF-36 & $1.5 I^{* *}(\mathrm{I} .0 \mathrm{I}$ to 2.45$)$ & $-0.89 *(-1.35$ to 1.34$)$ & $-1.40^{* *}(-1.90$ to 1.92$)$ & $1.34 * *(0.89$ to 1.85$)$ \\
\hline
\end{tabular}

I. All scales patient-rated. Score ranges: BSPS fear and avoidance sub-scales 0-28, somatic reaction sub-scale 0-16; FNE 0-30, MFQ 0-40, SDI 0-10, SF-35 0-100.

2. Negative values indicate deterioration.

$* P<0.05, * * P<0.01$.

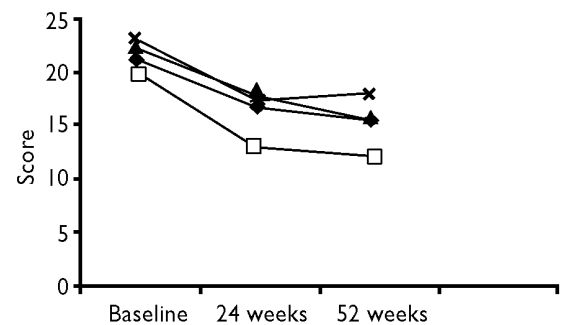

Fig. 3 Mean scores on the Fear of Negative Evaluation scale at baseline, week 24 and week 52 (range 0-30). $\diamond$, placebo; $\square$, sertraline + exposure; $\boldsymbol{\Delta}$, exposure; $\mathbf{x}$, sertraline

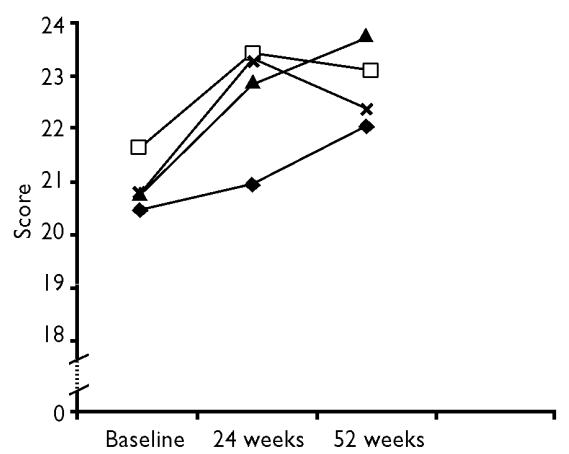

Fig. 4 Mean scores on the 36-item Short Form Health Survey at baseline, week 24 and week 52 (range 0-100). $\bullet$, placebo; $\square$, sertraline + exposure; $\boldsymbol{\Delta}$, exposure; $\mathbf{x}$, sertraline

change $-2.74,95 \%$ CI -4.51 to -0.97 , $P<0.01)$. The sertraline plus exposure group had a significant deterioration in SF-36 compared with both exposure-alone and placebo (mean difference in change
$-2.22,95 \%$ CI -4.02 to -0.43 , $P=0.01$, and $-2.40,95 \% \mathrm{CI}-4.17$ to $-0.63, P<0.01$, respectively). Changes in sertraline plus exposure compared with sertraline alone and in exposure alone compared with placebo were non-significant (Tables 3 and 4, Figs 3 and 4).

The sertraline plus exposure group had a significant deterioration in MADRS score $(P<0.01)$ between week 24 and week 52 . The changes in the other treatment groups were not significant.

\section{Treatment during the follow-up period}

Sixty-six patients $(20.5 \%)$ were treated with selective serotonin reuptake inhibitors (SSRIs): $13(15.5 \%)$ in the combined sertraline plus exposure group, $18(21.6 \%)$ in the sertraline-alone group, $14(19.2 \%)$ in the exposure group and $21(19.5 \%)$ in the placebo group. Twenty-seven patients $(7.6 \%)$ were offered exposure therapy by their general practitioner during the follow-up period, and 26 patients $(7.0 \%)$ had been referred to a psychologist or psychiatrist.

\section{Psychiatric diagnoses at week 52}

A total of 19 patients $(5.7 \%)$ had a major depression according to DSM-IV at week 52. Ten of these patients were in the group who had received the combined sertraline and exposure therapy, 2 had received sertraline alone, 4 had received exposure alone and 3 were in the placebo group.

\section{Employment records}

At the week 52 assessment 223 patients were employed and 33 were students. About a third $(32 \%)$ of the patients were on sick leave the year preceding baseline; less than a quarter $(23 \%)$ were on sick leave the year preceding follow-up. There was also a slight reduction in mean days of sick leave: 15.8 days in the year preceding baseline and 13.0 days in the year preceding follow-up.

\section{DISCUSSION}

This is, to our knowledge, the largest follow-up study of the treatment of social phobia, covering 375 patients. The study was carried out in primary care by a total of 45 physicians treating about 8 patients each. The patients were well known to the doctors and this provided an opportunity to make follow-up assessments of nearly all patients. Only 18 patients were lost to follow-up between weeks 24 and 52 .

\section{Maintenance of treatment effect}

All four treatment groups had a significant improvement from baseline to week 52 on all the psychometric assessments. Patients who had been given exposure therapy or placebo had a further improvement in 

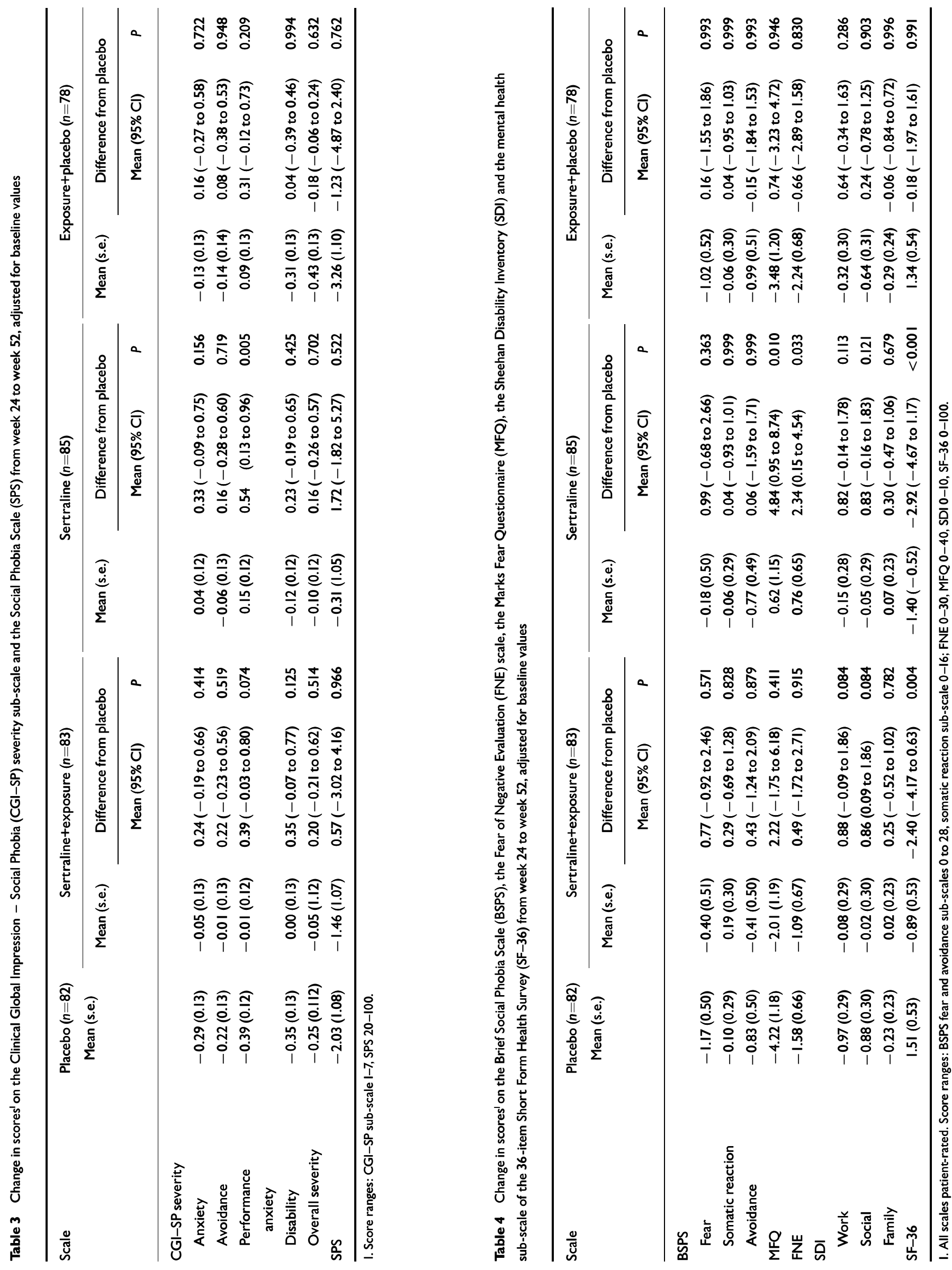
social phobia after the end of the treatment period, whereas patients who had been treated with sertraline - either alone or in combination with exposure therapy - had no further improvement during the follow-up period and there was a tendency towards deterioration. However, the deterioration was significant only for the score on SF-36 (Table 2). At week 52 the participants in both the sertraline-alone and the combined sertraline plus exposure groups had a significant deterioration compared with patients in the exposure-alone and placebo groups. These results are in accordance with the study by Liebowitz $e t$ al (1999), who concluded that cognitivebehavioural group therapy and phenelzine differed in their long-term effects, with a more favourable outcome for the group therapy. They also accord with the findings by Marks et al (1993), in a multi-centre study of alprazolam and exposure therapy in panic disorder, that gains after alprazolam were lost during follow-up, whereas gains after exposure were maintained. Combining alprazolam with exposure marginally enhanced gains during treatment, but impaired improvement thereafter. Barlow et al (2000), in a study of imipramine and cognitive-behavioural therapy in the treatment of panic, reported similar results. From this we can conclude that exposure techniques applied in situations with low levels of anxiety achieved by medication may have less impact than exposure therapy applied in situations with a higher level of anxiety, and may lead to a higher degree of relapse after end of treatment. During the follow-up period about a fifth of the patients were treated with SSRIs, $25(7.5 \%)$ were given exposure therapy by their general practitioner and $23(7.0 \%)$ were referred to psychiatrists or psychologists. The treatment was initiated at the discretion of the general practitioners, so we lack information about whether the treatment was given because of insufficient improvement at week 24 or because of relapse. However, this additional treatment might have contributed to the maintenance of treatment effect during the follow-up period.

\section{General effects}

A substantial proportion of the patients improved regardless of the treatment given, and even in the placebo-alone group only about a fifth of the patients were in need of additional treatment during the followup period. The fact that social phobia was

\section{CLINICAL IMPLICATIONS}

Sertraline, exposure therapy, and combined treatment with sertraline and exposure therapy are all effective treatments for social phobia.

- Treatment with exposure therapy alone seems to give further improvement subsequently, whereas patients treated with sertraline show a tendency towards deterioration after cessation of medication.

- Exposure therapy given alone is more effective in the long term than when given in combination with sertraline.

\section{LIMITATIONS}

- The general practitioners evaluated their own patients at both week 24 and week 52.

- There was no waiting-list control group in the study.

- There was no systematic registration of relapses during follow-up.

TONE TANGEN HAUG, MD, Haukeland University Hospital, Bergen; SVEIN BLOMHOFF, MD, National Hospital, Oslo, Norway; KERSTIN HELLSTRØM, DrMedSci, Ulleråker University Hospital, Uppsala, Sweden; INGAR HOLME, PhD, Ullevål University Hospital, Norway; MATS HUMBLE, MD, Huddinge University Hospital, Sweden; HANS PETTER MADSBU, MD, Elverum Medical Center, Elverum; JAN EGIL WOLD, MD, Innherred Hospital, Levanger, Norway

Correspondence: Tone Tangen Haug, Haukeland University Hospital, 002 I Bergen, Norway

(First received 24 April 2002, final revision 15 August 2002, accepted 23 October 2002)

focused on as a problem, combined with regular contact with a general practitioner in a total of 11 sessions over 24 weeks, seemed to be sufficient treatment for many patients.

\section{Methodological considerations}

The general practitioners evaluated their own patients at both week 24 and week 52. This lack of masking may represent a potential bias. Since many of these doctors worked in single-handed practices, it was difficult to obtain masked efficacy measures. However, scores on social phobia were achieved both on investigatorrated CGI-SP overall severity and on patient-rated SPS.

To evaluate the specific effects of exposure therapy and sertraline, a waiting-list control group could have been useful. In addition, this study does not inform us of follow-up periods longer than 28 weeks. There was no systematic registration of relapses during follow-up and the physicians initiated additional treatment based on their own clinical judgement. This limits our possibility to draw firm conclusions about maintenance therapy and relapse prevention. However, the study was naturalistic and was conducted in general practice, where most patients with social phobia will have their treatment. The study also had a large sample size, which strengthens the statistical power.

\section{Which treatment should be chosen for social phobia?}

Exposure therapy, sertraline and the combination of sertraline and exposure therapy are all effective treatments for social phobia. Sertraline and the combination of sertraline and exposure seemed to have a short-term advantage, but gains were reduced during follow-up. For exposure therapy alone there seemed to be a further improvement after the end of active treatment, and there are indications that exposure therapy alone is more effective in the long term than exposure in combination with sertraline treatment. For some 
patients, just being in regular contact with a general practitioner focusing on social phobia as a problem is effective.

\section{REFERENCES}

American Psychiatric Association (1994) Diagnostic and Statistical Manual of Mental Disorders (4th edn) (DSM-IV). Washington, DC: APA.

Barlow, D. H., Gorman, J. M., Shear, M. K., et al (2000) Cognitive-behavioral therapy, imipramine, or their combination for panic disorder: a randomized controlled trial. JAMA, 283, 2529-2536.

Blomhoff, S., Haug, T. T., Hellström, K., et al (200I) Randomised controlled general practice trial of sertraline, exposure therapy and combined treatment in generalised social phobia. British Journal of Psychiatry, 179, 23-30.

Davidson, J. R., Potts, N. L., Richie, E. A., et al (199I) The Brief Social Phobia Scale. Journal of Clinical Psychiatry, 52 (suppl. II), 48-5|.

_ , Potts, N. L. S., Richichi, E. A. (1993) Treatment of social phobia with clonazepam and placebo. Journal of Clinical Psychopharmacology, 13, 423-428.

Gelernter, C. S., Uhde, T.W., Cimbolic, P., et al (1991) Cognitive behavioral and pharmacological treatments of social phobia. Archives of General Psychiatry, 48, 938-945.

Haug, T.T., Blomhoff, S., Hellstrøm, K., et al (2000) The treatment of social phobia in general practice. Is exposure therapy feasible? Family Practice, 17, |14-118.

Heimberg, R. G., Salzman, D., Holt, C., et al (1993) Cognitive behavioral group treatment for social phobia: comparison with a credible placebo control. Cognitive

Therapy and Research, 14, I-23.

Liebowitz, M. R., Hope, D. A., et al (1998)

Cognitive-behavioral group therapy vs. phenelzine therapy for social phobia. Archives of General Psychiatry, 55, $\mid 133-1141$.

Katzelnick, D. J., Kobak, K. A., Greist, J. H., et al (1995) Sertraline for social phobia: a double blind, placebo-controlled crossover study. American Journal of Psychiatry, I52, |368-137|.

Leon, A. C., Shear, M. K., Portera, L., et al (1992) Assessing impairment in patients with panic disorder: the Sheehan Disability Scale. Social and Psychiatric Epidemiology, 27, 78-82.

Liebowitz, M. R., Schneier, F. R., Campeas, R., et al (1992) Phenelzine vs. atenolol in social phobia. A placebo controlled experiment. Archives of General Psychiatry, 49 290-300.

_, Heimberg, R. G., Schneier, F., et al (1999) Cognitive-behavioral group therapy versus phenelzine in social phobia: long term outcome. Depression and Anxiety, 10, 89-98.

Marks, I. M. \& Matthews, A. N. (1979) Brief standard self-rating for phobic patients. Behavior Research and Therapy, I7, 263-267.

, Swinson, R. P., Basoglu, M., et al (1993)

Alprazolam and exposure alone and combined in panic disorder with agoraphobia. A controlled study in London and Toronto. British Journal of Psychiatry, 162 , 776-787.

Mattick, R. P. \& Clarke, J. C. (1998) Development and validation of measures of social phobia scrutiny fear and social interaction anxiety. Behaviour Research and Therapy, 36, 455-470.

_ , Peters, L. \& Clark, J. C. (1989) Exposure and cognitive restructuring for severe social phobia. Behavior Therapy, 20, 3-23.

McHorney, C. A., Ware, J. E. \& Raczek, A. E. (1993) The MOS 36 Short Form Health Survey (SF-36): II. Psychometric and clinical tests of validity in measuring physical and mental health constructs. Medical Care, 3I 247-263.
Montgomery, S. A. \& Åsberg, M. (1979) A new depression scale designed to be sensitive to change. British Journal of Psychiatry, I34, 382-389.

SAS Institute (1997) SAS/STAT Software: Changes and Enhancements through Release 6.12. Cary, NC: SAS Institute.

Scholing, A. \& Emmelkamp, P. M. G. (1999) Prediction of treatment outcome in social phobia: a cross validation. Behaviour Research and Therapy, 37 $659-670$.

Sheehan, D., Lecrubier, Y., Janavs, J., et al (1994) International Neuropsychiatric Interview (MINI). Tampa, FL: University of South Florida Institute for Research in Psychiatry/INSERM-Hospital de la Salpêtrière.

Stein, M. B., Liebowitz, M. T., Lydiard, R. B., et al (1998) Paroxetine treatment of generalized social phobia (social anxiety disorder). JAMA, 280, 708-731.

Taylor, S. (1996) Meta-analysis of cognitive behaviora treatments of social phobia. Journal of Behavioral and Experimental Psychiatry, 27, I-9.

Turner, S., Breidel, D. \& Jacob, R. (1994) Social phobia: a comparison of behavior therapy and atenolol. Journal of Consulting and Clinical Psychology, 62, 350-358.

Van Vlet, I. M., den Boer, J. A. \& Westenberg, H. G. M. (1994) Psychopharmacological treatment of social phobia; a double blind placebo controlled study with fluvoxamine. Psychopharmacology, II5, 128-134.

Versiani, M., Nardi, A. E., Mundim, F. D., et al (1992) Pharmacotherapy of social phobia: a controlled study with moclobemide and phenelzine. British Journal of Psychiatry, I6I, 353-360.

Watson, D. \& Friend, R. (1969) Measurement of social evaluative anxiety. Journal of Consulting and Clinical Psychology, 31, 448-457. 